\title{
Metformin Protects Renal Function in Type 2 Diabetic Nephropathy: Regulation of Insulin Signalling in the Rat Model
}

\author{
C. WANG AND CHEN QIAO*
}

School of Basic Medicine and Clinical Pharmacy, China Pharmaceutical University, No.639 Longmian Road, Jiangning District, Nanjing, Jiangsu, China

\section{Wang and Qiao: Metformin Protect Renal Function in Type 2 Diabetic Nephropathy}

\begin{abstract}
The present study was aimed to investigate the protective role of metformin in type $\mathbf{2}$ diabetic nephropathic rats and elucidate regulation of unwanted insulin signalling in the kidney as the underlying mechanism. Diabetes mellitus was established in male Sprague Dawley rats by a single intraperitoneal injection of $40 \mathrm{mg} / \mathrm{kg}$ streptozotocin. A sustained level of blood glucose over $16.7 \mathrm{mM}$ was defined as type 2 diabetes mellitus in this study. Rats were randomly classified into the normal group, diabetes mellitus group, diabetes mellitus plus low-dose and high-dose metformin groups $(100$ and $200 \mathrm{mg} / \mathrm{kg}$ orally). Rats received treatment for 12 weeks after which all rats were sacrificed and kidney samples were collected. The samples were dissected and prepared for the observation of structural changes of renal glomerular podocytes between the groups microscopically. The expressions of IRS-1, Akt, nephrin, desmin, phosphatidylinositol3-hydroxy kinase (PI3K)-p85 and extra cellular matrix were probed by western blot. Compared to the control group, the downregulated nephrin and elevated levels of desmin and collagen IV in the kidney were significantly reversed by metformin treatment. Furthermore, metformin recovered the atypical activities of insulin/PI3K-Akt signalling to the normal ranges. Metformin evidently reduced the renal damage in diabetic rats, which could be due to modulating lipid metabolism and insulin resistance.
\end{abstract}

Key words: Metformin, type 2 diabetic nephropathy, insulin resistance, insulin signalling

Diabetes mellitus (DM) is a long-term hyperglycemia syndrome due to lack of insulin or insulin resistance. Diabetic nephropathy (DN) that resulting from DMinduced kidney damage is one of the most common and most serious complications of diabetes ${ }^{[1]}$. Microalbuminuria, the first indication of $\mathrm{DN}$ in patients, further progresses during $\mathrm{DN}$ because of damaged glomerular filtration to high levels of proteinuria ${ }^{[2]}$. So far, no drug has been successfully developed for the treatment of DN.

As type 2 diabetes mellitus (T2DM) patients account for the vast majority of those with diabetes, DN is the most common secondary disease, while correction of insulin resistance is critical to its improvement ${ }^{[3]}$. The abnormality of glomerular filtration barrier is one of the salient features of type $2 \mathrm{DN}$ (T2DN). Podocytes, as the most important component of glomerular filtration barrier in the outermost layer of glomerular membrane, control glomerular filtration through the diaphragm

*Address for correspondence

E-mail: 279201754@qq.com

May-June 2020 perforated by primary and secondary foot formation. Therefore, structural damage would result in loss of glomerular filtration barrier and ultimately lead to proteinuria. Previous reports have primarily proved that podocytes in the kidney are insulin-sensitive ${ }^{[4,5]}$. In T2DM, the disorder of insulin signaling would bring about the dysfunction of foot movement regulated by podocytes. In the progress of $\mathrm{DN}$, there is renal fibrosis caused by hyperplasia of glomerular mesangial cells, excessive synthesis of extracellular matrix (ECM) and attenuative gelatinase activity of decomposing $\mathrm{ECM}^{[6,7]}$. It has been reported that insulin receptors (IR) also exist in mesangial cells ${ }^{[8]}$. In vitro studies have shown that insulin can act on glomerular mesangial

This is an open access article distributed under the terms of the Creative Commons Attribution-NonCommercial-ShareAlike 3.0 License, which allows others to remix, tweak, and build upon the work non-commercially, as long as the author is credited and the new creations are licensed under the identical terms

Accepted 03 April 2020

Revised 29 December 2019

Received 03 December 2019 Indian J Pharm Sci 2020;82(3):522-527 
cells to enhance the activity of gelatinase through ERK and PI3K signaling pathways, which greatly promote the degradation of excessive $\mathrm{ECM}^{[9,10]}$. Therefore, the amelioration of insulin resistance in the kidney is the key section to the treatment of T2DM.

Metformin presents the effects of repressing lipid metabolism disorders and insulin resistance. It is reported that metformin has a significant therapeutic effect on atherosclerosis in hypercholesterolemic rats. Glucose tolerance test and insulin tolerance test in mice indicated that metformin can markedly promote the hypoglycemic effect of insulin. Furthermore, metformin has a certain improvement effect on lipid metabolism and enhancement of insulin sensitivity to peroxisome proliferator-activated receptor- $\alpha$ in mouse liver in a dose-dependent manner. Previous studies have shown that metformin has efficiently improved lipid metabolic disorder in DM by facilitating the expressions of genes related to lipid metabolism in mouse liver ${ }^{[11,12]}$. It has been further demonstrated that metformin can meliorate insulin resistance in muscle cells, ameliorating the phosphorylation of IRS-1 and up-regulating the downstream protein level of PI3 $\mathrm{K}^{[13]}$.

Considering the reparative effects of metformin on lipid metabolism disorder and insulin resistance, it was hypothesized that metformin might play an ameliorative role in renal deterioration in $\mathrm{T} 2 \mathrm{DN}$ rats. Therefore, this study aimed to explore the effect of metformin in renal dysfunction in T2DN rats and the potential mechanism involved, which could lay the foundation for further development of efficacious drugs against DM.

Forty male SPF Sprague-Dawley rats (160-180 g) were procured from the Experimental Animal Center of Zhejiang Province (Hangzhou, China). All experimental protocols were implemented in conformity with the Guide for Care and Use of Laboratory Animals of the Chinese National Institutes of Health. Streptozotocin (STZ) was purchased from Sigma-Aldrich. Rats were housed in a room with $12 \mathrm{~h}$ dark-light cycle with free access to food and water. All applicable international, national, and/or institutional guidelines for the care and use of animals were followed.

The rats were randomly divided into 4 groups according to their serum creatinine (SCE) values, group 1 the control group (Control), group 2, the diabetic group (DM), group $3 \mathrm{DM}$ plus $100 \mathrm{mg} / \mathrm{kg} / \mathrm{d}$ metformin group (DM+Met-L) and group 4 DM plus $200 \mathrm{mg} / \mathrm{kg} / \mathrm{d}$ metformin group (DM+Met-H). Prior to the development of the model, rats of the four groups neither presented significant differences in blood glucose, SCE, blood urea nitrogen and urinary creatinine in intragroup comparisons, nor shown significance compared to the normal ones. After one week of adaptation, the T2DM model was established by a single injection of $40 \mathrm{mg} / \mathrm{kg} \mathrm{STZ}$ intraperitoneally. STZ was dissolved in citrate buffer solution $\mathrm{pH}$ 4.4. Blood glucose level $\geq 16.7 \mathrm{mM}$ after $72 \mathrm{~h}$ was considered as successful establishment of T2DM. All rats underwent $12-\mathrm{w}$ distinct treatments after grouping and modeling. All the experiments on rats were performed by competent person with qualification certification.

Formalin-fixed and paraffin-embedded kidney tissues were prepared and stained with $\mathrm{H}$ and $\mathrm{E}$ stain, Masson's trichrome stain and oil red O stain. Collagen area per visual field was analyzed by Image-Pro Plus 6.0 software. The pathological observation of kidney and electron microscopic observation of renal cortex were performed at the end of the study.

Total protein was extracted from the renal tissues and quantified by Western blot. Several bioactive substances in therenal membrane were probed with rabbit polyclonal transforming growth factor (TGF- $\beta$ ) antibody, rabbit polyclonal Smad2/3 antibody, rabbit monoclonal phosphor-Smad2 antibody, rabbit monoclonal phosphor-Smad3 antibody, rabbit polyclonal ERK1/2 antibody, rabbit monoclonal phosphor-ERK1/2 antibody (Cell Signaling Technology), rabbit polyclonal Collagen I antibody and rabbit polyclonal collagen III antibody (Abcam). Target proteins were visualized using enhanced chemiluminescence-detection reagents (Amersham Pharmacia Biotech). Protein loading was normalized to rabbit polyclonal $\beta$-actin antibody (CMC Scientific, Shanghai, China) and goat antirabbit secondary antibody (Abmart, Shanghai, China).

Results are presented as mean \pm SD. Data of multi-group comparisons were analyzed using one-way ANOVA with LSD post hoc test in SPSS 19.0 software. Data were plotted using GraphPad Prism 5 software. $P$ value less than 0.05 was accepted as statistically significant.

HE staining under microscope manifested glomerular hypertrophy, hyalinization of peripheral renal tubules and expansion of mesangium in the kidney samples. After 12-w treatment with metformin, the pathology of diabetic kidneys was significantly reversed. The renal cortical lipid accumulation index calculated by Image $J$ in DM group climbed up to 25.79 folds compared to that in the control group. The index in DM+Met-L group was 15.56 fold greater than that in the control group. The indices of DM+Met-H group was 10.05 fold and 10.84 fold higher than that of the control group, respectively. 
Glomerular basement membrane thickness (GBMT) was measured under electronic microscope with Medical Image System. Microscopically, foot process of glomerular podocytes disappeared or significantly fused in $12 \mathrm{w}$ T2DN rats, whereas administration of metformin ameliorated the injury of foot process and at least retained the morphological integrity of a portion of the glomerulus, the GBMT also reduced by metformin treatment (fig. 1).

Type IV collagen is mainly distributed in the ECM, forming the critical structure of glomerular basement membrane and mesangial matrix. Type I collagen expresses only in the renal interstitial and major blood-vessel wall but not in glomerular, and is proactively involved in inflammation, hardening and fibrosis. Consistent with the results of the Masson's trichrome staining, collagen I and IV and fibronectin were sporadically stained in glomeruli and glomeruli interstitial cells of the normal renal tissue. However, the levels of the three were up-regulated in the renal tissues of DM rats, which were completely overturned by metformin $(p<0.05)$. As shown in fig. 2 , the western blot analysis presented that the level of nephrin in the podocytes of DM rats declined compared to the control group. Metformin prevented these effects on podocytes by rectifying the anomaly of nephrin and desmin, the

\section{A}
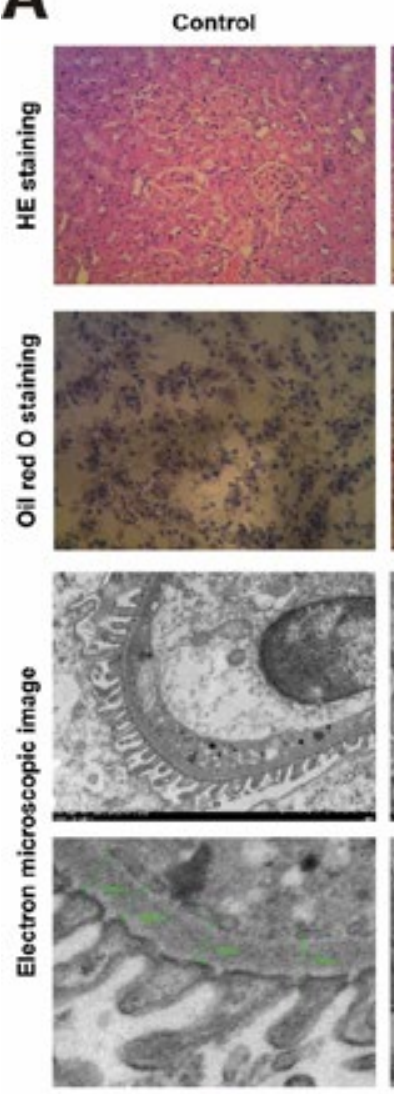

B

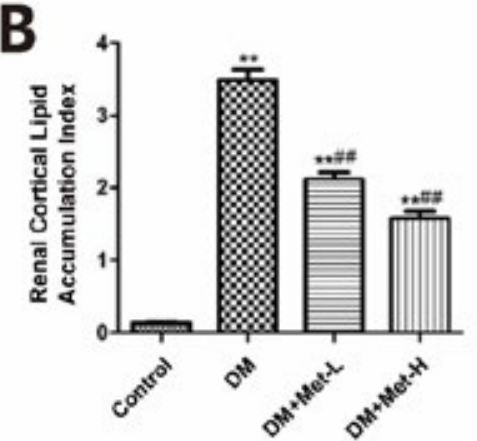

DM
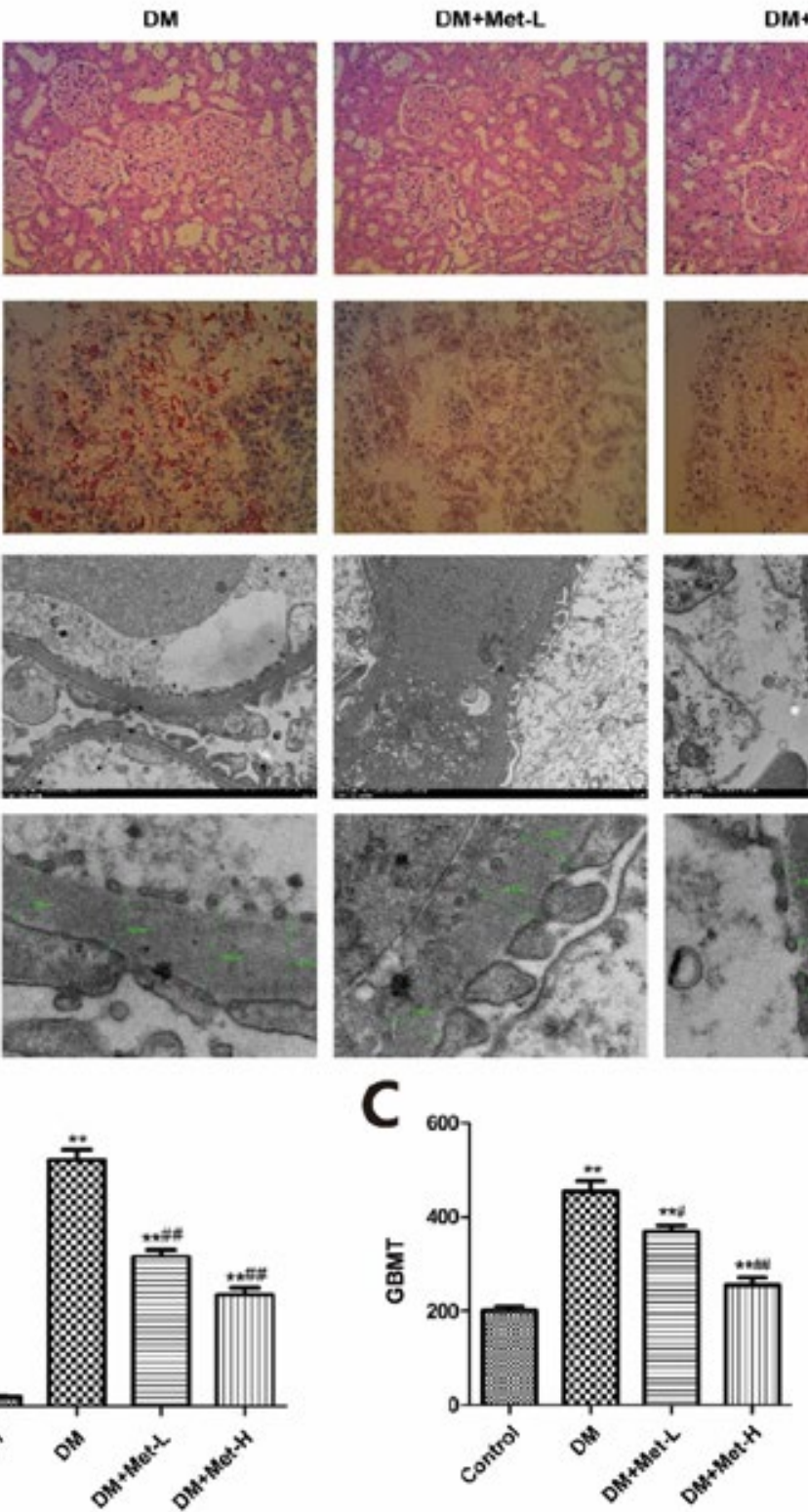

Fig. 1: Metformin mitigated pathology of diabetic kidney

A. HE staining and Oil red $O$ staining, electronic microscopic scan of glomerular podocytes, B. quantitative analysis of renal cortical lipid accumulation index, $C$. quantitative analysis of glomerular basement membrane thickness. Results are expressed as means \pm SEM. The data are presented as \% cell inhibition rate to unstimulated group 
markers of mesenchymal and epithelial cell damage, respectively.

PI3K-Akt signaling is involved in the underlying mechanism of DN. Therefore, it was investigated to see how metformin affected the PI3K-Akt signaling pathways. Furthermore, as shown in fig. 3, the phosphorylation levels of PI3K-p85 and Akt were elevated in DM+Met groups after 12-w of treatment. The signal transduction of p-IRS-1Ser307 was upregulated but that of p-IRS-1Tyr895 was downregulated in T2DN rats. This could be attributed to insulin resistance, resulting in improper translocation of GLUT-4 vesicles to the plasma membrane and disruption of glucose uptake thereby. Hence, the result suggested that metformin directly regulated abnormal IRS-1 phosphorylation, improving transduction of insulin signaling in renal tissue, and might be more promising than metformin.

The current research indicated that metformin exerted a protective effect on the renal function in $\mathrm{T} 2 \mathrm{DN}$ rats. The latest pharmacological research has shown that metformin has a remedial effect on lipid metabolism

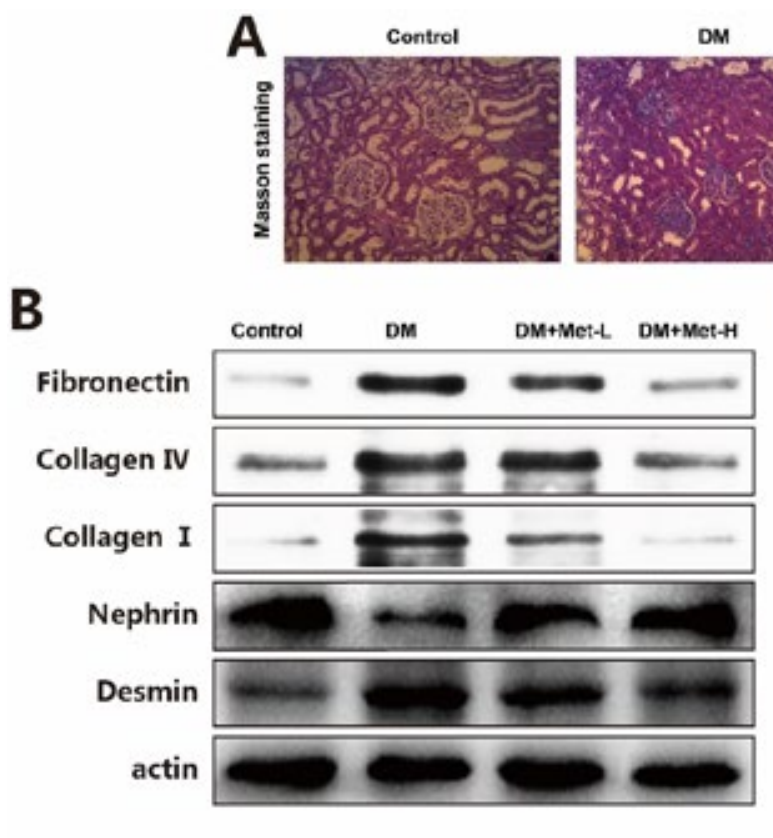

Fig. 2: Metformin decreased excessive accumulation of extracellular matrix in diabetic kidney
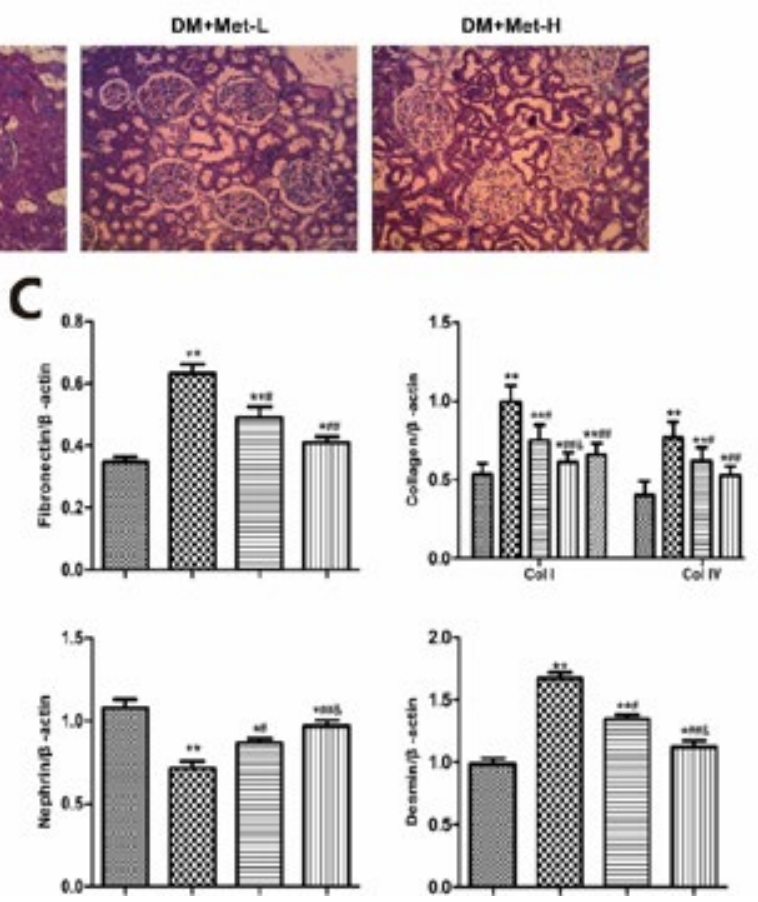

A. Masson's trichrome staining of glomerular podocytes, B. fibronectin, collagen I and IV, nephrin and desmin expression were

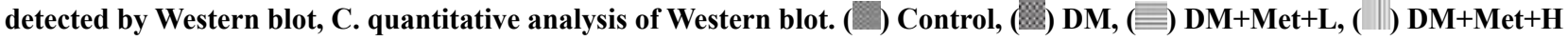
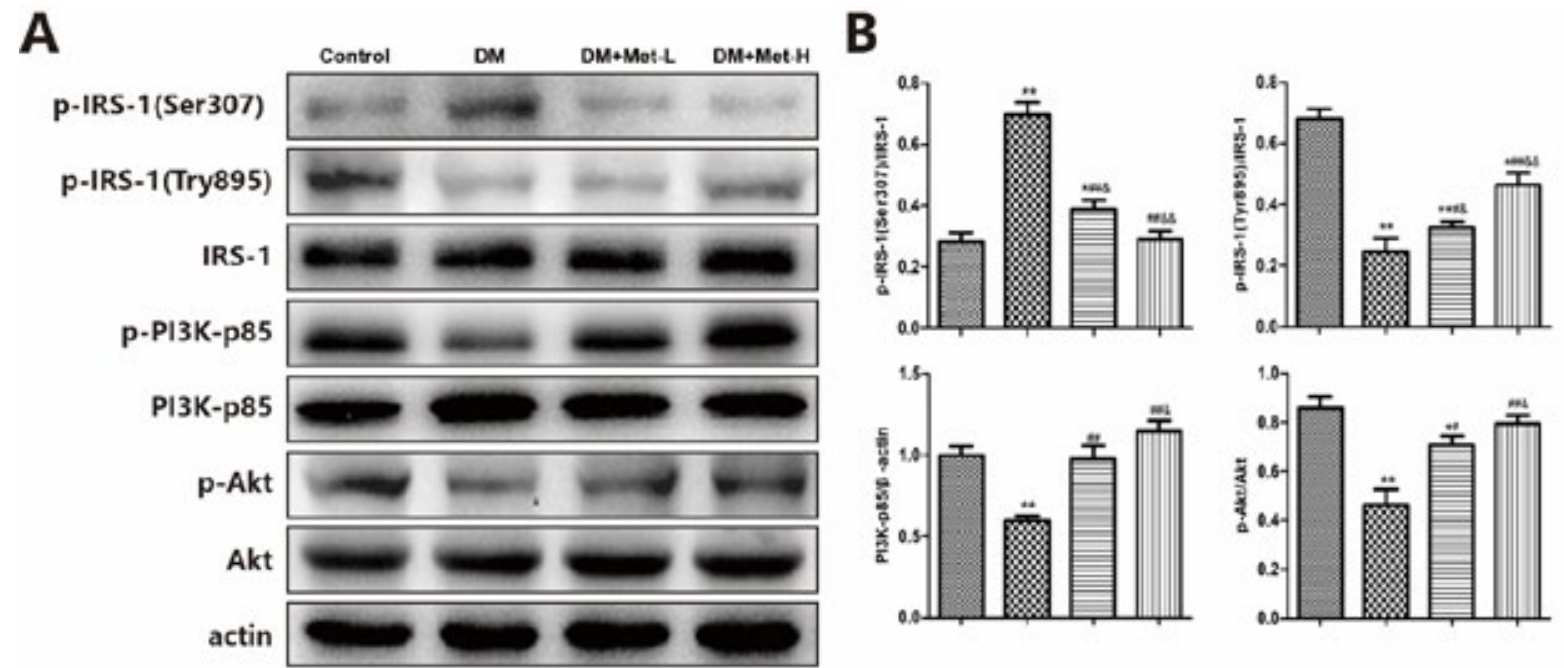

Fig. 3: Metformin normalized insulin / PI3K-Akt signaling

A. IRS-1, PI3K, Akt and their phosphoprotein expression as detected by Western blot, B. quantitative analysis of Western blot. (i) Control, (娄) DM, (春) DM+Met+L, (III) DM+Met+H 
disorder and insulin resistance. It was observed that the deviant phosphorylation level of IRS- 1 is the critical link bringing about insulin resistance in T2DN. As insulin resistance occurs, the tyrosine phosphorylation of IRS-1 is about to decline while the inhibitory serine phosphorylation of IRS-1 to increase, thus significantly suppressing the downstream intracellular pathways, comprising at least two pivotal ones, PI3K/ Akt signaling and mitogen-activated protein kinases signaling. Metformin could directly regulate the irregular phosphorylation level of IRS-1, manifesting its superiority to melbine over the improvement of insulin signal transduction in the kidney.

It is known that insulin resistance causes kidney fibrosis ${ }^{[14,15]}$. As the impacts of metformin on insulin resistance has been further verified in this study, metformin has alleviated ECM accumulation by repressing the expressions of collagen and fibronectin in mesangial cells. Besides, insulin also magnifies the activity of gelatinase by provoking PI3K signaling pathway in mesangial cells, dramatically promoting excessive ECM degradation and lightening glomerular fibrosis. Nevertheless, the effects of metformin are distinct among its species. In glomerular membrane cells, the declined expression of fibronectin by metformin may attribute to its restraint on the synthesis of TGF- $\beta 1$.

The incidence of abnormal function of glomerular filtration in DN results from unusual motion of foot process and atypical structure of foot process in podocytes. Therefore, the impacts of metformin on the structure and motion state of glomerular podocytes have been explored in this study. Metformin improves insulin resistance in the kidney via IRS-1/PI3K/AKTsignaling pathway, causallyincreasing the protein expression of nephrin. As is known to be a kind of transmembrane protein specifically existing in podocytes, nephrin is responsible for maintaining the structure of podocytes ${ }^{[16]}$. Moreover, insulin can even inhibit the expression of cytoskeletal silk protein desmin via IRS-1/PI3K/AKT pathways, suggesting that metformin can repress the skeleton realignment of podocytes, followed by foot process fusion and fading away and then relieving podocyte injury and phenotype transfusion. Therefore, metformin could improve the abnormality in glomerular filtration barrier by recovering the structure and motion state of glomerular podocyte.

The present study succinctly explained how metformin improved the impaired renal function in DN animals, namely provoking renal insulin signaling pathway. As unexpected factors might have interacted with and influenced each other on the overall level, causing unpredictable interference to the outcomes of the experiment, the detailed and inerrant mechanism still remains unclear.

In conclusion, metformin can improve the impairment of kidney in T2DN rat models established by high-glucose-high-fat diet and low-dose STZ. Metformin rectified the abnormal transduction of PI3K-Akt signaling by increasing the tyrosine phosphorylation level of IRS1 and reducing the inhibitory serine phosphorylation, causally improving the insulin resistance in the kidney. However, the accurate mechanism behind metformin's inhibition of collagen and fibronectin in mesangial cells still needs further exploration.

\section{REFERENCES}

1. El-Messallamy FAF, El-Ashmawy HM, El Shabrawy AM, Radwan SE. Proinsulin/insulin ratio as a predictor of insulin resistance in patients with diabetic nephropathy. Diabetes Metab Syndr 2019;13:2057-2060.

2. Thethi TK, Batuman V. Challenging the conventional wisdom on diabetic nephropathy: Is microalbuminuria the earliest event? J Diabetes Complications 2019;33:191-192.

3. Li Y, Li Q, Wang C, Lou Z, Li Q. Trigonelline reduced diabetic nephropathy and insulin resistance in type 2 diabetic rats through peroxisome proliferator-activated receptor-gamma. Exp Ther Med 2019;18:1331-37.

4. Lay AC, Coward RJM. The Evolving Importance of Insulin Signaling in Podocyte Health and Disease. Front Endocrinol 2018;9:693.

5. Zhan X, Yan C, Chen Y, Wei X, Xiao J, Deng L, et al. Celastrol antagonizes high glucose-evoked podocyte injury, inflammation and insulin resistance by restoring the HO-1mediated autophagy pathway. Mol Immunol 2018;104:61-8.

6. Yu FN, Hu ML, Wang XF, Li XP, Zang BH, Lu XQ, et al. Effects of microRNA-370 on mesangial cell proliferation and extracellular matrix accumulation by binding to canopy 1 in a rat model of diabetic nephropathy. J Cell Physiol 2019;234:6898-6907.

7. Zhou SX, Huo DM, He XY, Yu P, Xiao YH, Ou CL, et al. High glucose/lysophosphatidylcholine levels stimulate extracellular matrix deposition in diabetic nephropathy via plateletactivating factor receptor. Mol Med Rep 2018;17:2366-72.

8. Oemar BS, Foellmer HG, Hodgdon-Anandant L, Rosenzweig SA. Regulation of insulin-like growth factor I receptors in diabetic mesangial cells. J Biol Chem 1991;266:2369-73.

9. Li R, Wang T, Walia K, Gao B, Krepinsky JC. Regulation of profibrotic responses by ADAM17 activation in high glucose requires its C-terminus and FAK. J Cell Sci 2018;131:208629.

10. Park JT, Kato M, Yuan H, Castro N, Lanting L, Wang M, et al. FOG2 protein down-regulation by transforming growth factor-beta1-induced microRNA-200b/c leads to Akt kinase activation and glomerular mesangial hypertrophy related to diabetic nephropathy. J Biol Chem 2013;288:22469-480. 
11. Bai F, Liu Y, Tu T, Li B, Xiao Y, Ma Y, et al. Metformin regulates lipid metabolism in a canine model of atrial fibrillation through AMPK/PPAR-alpha/VLCAD pathway. Lipids Health Dis 2019;18:109.

12. Wang Z, Zhou J, Lu M, Liang Y, Jiang Z, Chen K, et al. Therapy of empagliflozin plus metformin on T2DM mice shows no higher amelioration for glucose and lipid metabolism than empagliflozin monotherapy. Life Sci 2019;232:116622.

13. Ertunc D, Tok EC, Aktas A, Erdal EM, Dilek S. The importance of IRS-1 Gly972Arg polymorphism in evaluating the response to metformin treatment in polycystic ovary syndrome. Hum Reprod 2005;20:1207-12.
14. Revuelto Artigas T, Velasco NZ, Arbones XG. Chronic hepatitis C infection: An independent risk factor for subclinical atheromatosis. Rev Clin Esp 2019;219:293-302.

15. Yoon JJ, Park JH, Kim HJ, Jin HG, Kim HY, AhnYM, et al. Dianthus superbus Improves Glomerular Fibrosis and Renal Dysfunction in Diabetic Nephropathy Model. Nutrients 2019;11:553.

16. Chen Z, Zhang Y, Zhao X. FAM40A alters the cytoskeleton of podocytes in familial focal and segmental glomerulosclerosis by regulating F-actin and nephrin. Arch Med Sci 2019;15:16573. 